\title{
Attenuated endothelial function is associated with decreased endothelial progenitor cells and nitric oxide in premenopausal diabetic women
}

\author{
JUAN LIU ${ }^{1 *}$, DA-JUN HU ${ }^{2,3^{*}}$, HUIYANG YAN ${ }^{4}$, JIANBIN LIU $^{5,6}, \mathrm{XIXONG} \mathrm{AI}^{7}$, \\ ZI REN ${ }^{7}$, HAITAO ZENG ${ }^{7}, \mathrm{HAO} \mathrm{HE}^{8}$ and ZHEN YANG ${ }^{9-12}$
}

${ }^{1}$ Department of Endocrinology, The First Affiliated Hospital, Sun Yat-Sen University, Guangzhou, Guangdong 510080;

${ }^{2}$ Department of Cardiology, The First Hospital of Chenzhou, Chenzhou, Hunan 423000;

${ }^{3}$ Department of Geriatric Medicine, Xiangya Hospital, Central South University, Changsha, Hunan 410008;

${ }^{4}$ Guangzhou Beijing Community Health Service Center, Guangzhou, Guangdong 510080, P.R. China;

${ }^{5}$ Centre for Eye Research Australia, Faculty of Medicine, Dentistry and Health Science, The University of Melbourne,

East Melbourne, VIC 3002; ${ }^{6}$ Department of General Medicine, Box Hill Hospital, Box Hill, VIC 3128, Australia;

${ }^{7}$ Center for Reproductive Medicine, The Sixth Affiliated Hospital, Sun Yat-Sen University, Guangzhou, Guangdong 510080;

${ }^{8}$ Department of Cardiology, The Fifth Affiliated Hospital of Zunyi Medical University, Zhuhai, Guangdong 519100;

${ }^{9}$ Division of Emergency Medicine; ${ }^{10}$ Emergency Intensive Care Unit; ${ }^{11}$ Department of General Internal Medicine,

The First Affiliated Hospital, Sun Yat-Sen University; ${ }^{12}$ Department of Cardiology and Key Laboratory on Assisted Circulation,

Ministry of Health, The First Affiliated Hospital, Sun Yat-Sen University, Guangzhou, Guangdong 510080, P.R. China

Received August 11, 2017; Accepted June 4, 2018

DOI: $10.3892 / \mathrm{mmr} .2018 .9451$

\begin{abstract}
Previous studies have demonstrated that the deleterious effect of diabetes mellitus (DM) on the risk of cardiovascular disease also occurs in premenopausal women, in spite of their relatively high estrogen levels; however, the underlying mechanism remains unclear. The present study aimed to investigate the sex-related differences in circulating endothelial progenitor cells (EPCs) in a relatively young population with type 2 DM (T2DM) and its underlying mechanism. The number and functional activity of circulating EPCs, and vascular endothelial function assessed using flow-mediated dilation (FMD), were compared in premenopausal women and age-matched men with or without T2DM. Nitric oxide (NO)
\end{abstract}

Correspondence to: Professor Zhen Yang, Department of Cardiology and Key Laboratory on Assisted Circulation, Ministry of Health, The First Affiliated Hospital, Sun Yat-Sen University, 58 Zhongshan 2nd Road, Guangzhou, Guangdong 510080, P.R. China E-mail: yangzhen10710710@163.com

Professor Hao He, Department of Cardiology, The Fifth Affiliated Hospital of Zunyi Medical University, 1439 Zhufeng Da Road, Zhuhai, Guangdong 519100, P.R. China

E-mail: hehao2003050@126.com

*Contributed equally

Key words: endothelial progenitor cells, premenopausal women, nitric oxide, endothelial function in the plasma or NO secreted by EPCs was also measured. The number and activity of circulating EPCs, and NO levels in the plasma or culture medium, were lower in premenopausal women with T2DM compared with those without T2DM. In addition, the number and activity of circulating EPCs and NO levels were decreased in men with T2DM compared with in age-matched premenopausal women with T2DM. FMD was positively correlated with the number and activity of circulating EPCs, and NO levels. In conclusion, DM in premenopausal women may significantly impair the repair capability of EPCs and lead to endothelial dysfunction, which may be associated with reduced NO production. In patients with both DM and normal glucose tolerance, sex-related differences of EPCs are presented in a young population.

\section{Introduction}

In the last decade, China has experienced a substantial increase in the prevalence of type 2 diabetes mellitus (T2DM) (1), which is characterized by high morbidity and mortality, due to concomitant cardiovascular complications. It has previously been revealed that the increased cardiovascular risk in patients with DM is partly attributed to the occurrence of endothelial injury, which triggers and accelerates the pathogenesis of atherosclerotic vascular disease (2). In patients and animals with DM, preventing endothelial dysfunction helps maintain vascular homeostasis and diminishes the occurrence of DM-associated vascular events (3-5).

Circulating endothelial progenitor cells (EPCs), which are derived from the bone marrow, serve a crucial role in promoting neovascularization, repairing endothelial damage 
and improving endothelial function (6). Numerous risk factors of cardiovascular disease (CVD), including hypertension, obesity, dyslipidemia and smoking, are usually concomitant with a decreased number and activity of circulating EPCs, which may accelerate the pathophysiological process of endothelial injury (7-10). However, hyperglycemia appears to be negatively correlated with circulating EPCs compared with the aforementioned CVD factors. The reduced number and impaired activity of circulating EPCs can be observed in patients with T1DM (11) and T2DM (12). Furthermore, reduced levels of circulating EPCs are correlated not only with glycemic control (13), but also with severity of impaired glucose tolerance in subjects with or without T2DM (14). Although the underlying mechanism is yet to be fully determined, numerous pathophysiological mechanisms, including inflammation induced by hyperglycemia, insulin resistance, oxidative stress and deletion of nitric oxide (NO), could be involved in these EPC alterations in patients with DM (12,15-17).

Patients with T2DM have a two- to four-fold increased risk of CVD (18). In addition, the deleterious effects of DM on CVD risk also occur in premenopausal women, in spite of their relatively high estrogen levels (19). Our previous studies indicated that, although the number and activity of circulating EPCs is preserved in prehypertensive premenopausal women compared with in those without prehypertension (20), DM concomitantly diminishes their EPC advantages in terms of vascular protection, thus resulting in a reduced number and impaired activity of EPCs, and even damaged endothelial function (21). However, the following issues require further investigation: i) Whether attenuated endothelial function generally occurs in all premenopausal women with T2DM and ii) whether the difference in circulating EPCs between the sexes is influenced by the coexistence of DM. Certain factors resulting from plasma or cultured medium, including $\mathrm{NO}$, vascular endothelial growth factor (VEGF) and granulocyte macrophage-colony-stimulating factor (GM-CSF), are involved in modulating the number and activity of circulating EPCs (22-25); however, only NO is involved in modulating the sex-related differences in circulating EPCs in a prehypertensive population (20). The present study aimed to determine whether similar results may be present in the DM population. The present study evaluated the number and activity of circulating EPCs in women and men with DM and normal glucose tolerance (NGT); in addition, NO, VEGF, and GM-CSF levels in the human plasma, as well as in cultured EPC media, were measured to identify the possible underlying mechanisms.

\section{Materials and methods}

Characteristics of subjects. A total of 40 premenopausal women (20 with NGT and 20 with T2DM) and 40 age-matched men (20 with NGT and 20 with T2DM; aged 36-51 years old), were recruited to the present study between July 2015 and May 2017 in the First Hospital of Chenzhou and Xiangya Hospital, Central South University (Changsha, China). The enrolled patients with NGT were diagnosed with a fasting plasma glucose (FPG) of $\leq 5.6 \mathrm{mmol} / 1$ and 2-h plasma glucose (2-h PG) following oral glucose challenge of $\leq 7.8 \mathrm{mmol} / \mathrm{l}$; those with $\mathrm{T} 2 \mathrm{DM}$ were diagnosed with a FPG of $\geq 7.0 \mathrm{mmol} / \mathrm{l}$ and/or 2 -h PG of $\geq 11.1 \mathrm{mmol} / 1$ and/or glycated hemoglobin (HbA1c) of $\geq 6.5 \%$ based on the Experts Committee Reports of the American Diabetes Association (26). NGT subjects had no vascular risk factors, including obesity, hypertension, smoking and dyslipidemia. All subjects had no malignant disease, infection, inflammatory disorders, known CVD, or ongoing medical treatments (including with antiplatelet, anti-inflammatory or hypolipidemic agents). Nursing or pregnant women were excluded from the present study. The present study was approved by the Ethics committee of the First Hospital of Chenzhou and Xiangya Hospital, Central South University, and written informed consent was obtained from all participants.

Following an overnight fast for 8-12 h, 75 g glucose solution was administered orally within $5 \mathrm{~min}$. Blood samples $(5 \mathrm{ml})$ were collected prior to, and at 30 and $120 \mathrm{~min}$ post-challenge, and plasma glucose was measured.

FPG, HbA1c, total cholesterol (TC), triglycerides (TG), low- and high-density lipoprotein (LDL and HDL) cholesterol and estrogen levels were measured. Peripheral blood samples were collected to determine the number and activity of EPCs.

Flow cytometry and cell culture assay to assess the number of circulating EPCs. The primary circulating EPCs were applied to the following analysis. EPCs were assessed based on the described methods in our previous studies $(22,27,28)$. The peripheral blood mononuclear cells of the participants were isolated using Ficoll density gradient centrifugation, then suspended in endothelial cell growth medium $2(500 \mathrm{ml}$; Lonza Group, Ltd., Basel, Switzerland) supplemented with $2 \%$ fetal bovine serum (Sigma-Aldrich; Merck KGaA). The cell suspension $\left(2.5 \times 10^{6} / \mathrm{ml}\right)$ was added to cell culture flasks (25 cm²; Corning, Inc., Corning, NY, USA), coated with fibronectin (Clonetics Co., San Diego, CA, USA) and incubated at $37^{\circ} \mathrm{C}$ in a humidified environment containing $5 \% \mathrm{CO}_{2}$. After 4 days, nonadherent cells were removed and adherent cells were maintained for another 7 days; these cells were used for subsequent experiments.

Following 7 days of culture, endothelial marker proteins were examined by flow cytometry (29). Peripheral blood $(100 \mu \mathrm{l})$ was incubated for $40 \mathrm{~min}$ at $4^{\circ} \mathrm{C}$ with phycoerythrin (PE)-labeled monoclonal mouse anti-human antibodies recognizing cluster of differentiation (CD)31 (1:10; cat. no. 745290; BD Biosciences, San Jose, CA, USA), von Willebrand factor (1:10; cat. no. 555849; BD Biosciences) and kinase-insert domain receptor (KDR; 1:20; cat. no. FHK309-025; 4A Biotech, Co., Ltd., Beijing, China) or corresponding immunoglobulin G isotype control (1:10; cat. no. FMCK001K-025IU; 4A Biotech, Co., Ltd.). Following this, erythrocytes were lysed, and the remaining cells were washed with PBS and fixed in $2 \%$ paraformaldehyde at $37^{\circ} \mathrm{C}$ for $10 \mathrm{~min}$ prior to further analysis using a ACEA NovoCyte ${ }^{\mathrm{TM}}$ (ACEA Biosciences, San Diego, CA, USA). Cells were then incubated with monocytic lineage marker CD14 (1:10; cat. no. 555397; BD Biosciences), fluorescein isothiocyanate (FITC) anti-human CD45 (1:10; cat. no. FHF045-025; 4A Biotech, Co., Ltd.) and PE-Cy7 anti-human CD34 (1:10; cat. no. FHN034-025; 4A Biotech, Co., Ltd.) antibodies for $40 \mathrm{~min}$ at $4^{\circ} \mathrm{C}$. NovoExpress software $^{\text {TM }}$ (ACEA Biosciences, San Diego, CA, USA) was used to analyze the results. 
The ratio of $\mathrm{CD} 34^{+} \mathrm{KDR}^{+}$cells per 100 peripheral blood mononuclear cells was used to count the circulating EPCs. To confirm the EPC phenotype, mononuclear cells $\left(2.5 \times 10^{6} / \mathrm{ml}\right)$ were plated on cell culture flasks with EBM and after 7 days culture, the attached EC-like cells were incubated with 1,1'-dioctadecyl-3,3,3',3'-tetramethylindo-carbocyanine perchlorate-labeled acetylated LDL (DiI-acLDL; Molecular Probes; Thermo Fisher Scientific, Inc.) at $37^{\circ} \mathrm{C}$ for $1 \mathrm{~h}$. The cells were then fixed with $4 \%$ paraformaldehyde for $30 \mathrm{~min}$ at $37^{\circ} \mathrm{C}$ and incubated with FITC-labeled lectin (Sigma-Aldrich; Merck KGaA, Darmstadt, Germany) for $4 \mathrm{~h}$ at $37^{\circ} \mathrm{C}$. After staining, the samples were observed by two independent observers under a phase-contrast fluorescence microscope (magnification, x200). Cells demonstrating double-positive fluorescence were identified as differentiating EPCs.

Migration and proliferation assay of EPCs. EPC migration and proliferation assays were described in our previous studies $(22,27,28)$. Similar methods were adopted in the present study.

Following harvesting by centrifugation $\left(438 \mathrm{x} \mathrm{g}\right.$ at $4^{\circ} \mathrm{C}$ for $5 \mathrm{~min}$ ), EPCs were suspended in $500 \mu \mathrm{l} \mathrm{EBM}$ and migration was then analyzed. A total of $2 \times 10^{4}$ EPCs were placed in the upper chamber of a modified Boyden chamber, which was placed in a 24-well culture dish containing EBM and human recombinant VEGF (50 ng/ml). Following incubation at $37^{\circ} \mathrm{C}$ for $24 \mathrm{~h}$, the lower side of the filter was washed with PBS and fixed with $2 \%$ paraformaldehyde at $37^{\circ} \mathrm{C}$ for $10 \mathrm{~min}$. For quantification, cell nuclei were stained with DAPI. Cells migrating into the lower chamber were counted manually in three random fields using a fluorescence microscope.

The proliferation of EPCs was analyzed as follows. After culturing for 7 days, EPCs were digested using $0.25 \%$ trypsin, and were cultured in serum-free medium in 96-well culture plates (200 $\mu \mathrm{l} /$ well) for $24 \mathrm{~h}$. Subsequently, EPCs were supplemented with $10 \mu 1$ MTT (5 g/l; Fluka; Honeywell International, Inc., Shanghai, China) and incubated for a further $4 \mathrm{~h}$. The supernatant was discarded by aspiration and the EPC preparation was agitated with $200 \mu$ l dimethyl sulfoxide for $10 \mathrm{~min}$, prior to the measurement of optical density at $490 \mathrm{~nm}$.

Measurement of NO, VEGF and GM-CSF levels in the plasma and secreted by EPCs. Nitrite, the stable metabolite of $\mathrm{NO}$, was measured in plasma using the Greiss method, as described in our previous studies $(22,27-29)$. The results were presented as mmol $\mathrm{NO}_{\mathrm{x}}$ of $\mathrm{NO}_{3}{ }^{-} / \mathrm{NO}_{2}{ }^{-}$per liter of medium. High-sensitivity ELISA assays (VEGF, cat. no. DVE00; GM-CSF, cat. no. DGM00; R\&D Systems, Europe, Ltd., Abingdon, UK) were used to measure plasma levels (which were isolated from blood samples via centrifugation at $291 \mathrm{x} \mathrm{g}$ for $5 \mathrm{~min}$ at room temperature) of VEGF and GM-CSF in accordance with the manufacturer's protocol.

After transferring the cultured EPCs $\left(2 \times 10^{5} / \mathrm{ml}\right.$ cells for the determination NO and VEGF levels, and $1 \times 10^{5} / \mathrm{ml}$ cells for the determination of GM-CSF levels) to Dulbecco's modified Eagle's medium (Gibco; Thermo Fisher Scientific, Inc.)/20\% fetal bovine serum (Sigma-Aldrich; Merck KGaA) for $48 \mathrm{~h}$, NO, VEGF and GM-CSF levels were measured in the conditioned media, using the Griess method and ELISA assays according to the aforementioned protocol.
Measurement of flow-mediated dilation (FMD). As previously described $(30,31)$, higher solution ultrasonography using a 5-12 MHz linear transducer on an HDI 5,000 system (Philips Healthcare, Andover, MA, USA) was used to measure the brachial artery FMD. Participants were placed in a supine position, a sphygmomanometer cuff was placed at the brachial artery on the upper-forearm (20-100 $\mathrm{mm}$ proximal to the antecubital fossa) and the pressure was increased to $250 \mathrm{mmHg}$ for $5 \mathrm{~min}$. FMD was calculated as the percentage increase in mean diastolic diameter following a reactive hyperemia of 55-65 sec, followed by deflation to the baseline. After a further $15 \mathrm{~min}, 400 \mu \mathrm{g}$ sublingual glyceryltrinitrate was injected and the diastolic diameter was measured again after $5 \mathrm{~min}$ to determine endothelial-independent dilatation.

Statistical analysis. SPSS v11.0 (SPSS Inc., Chicago, IL, USA) was used for all statistical analyses. Quantitative variables with normal distribution were presented as the means \pm standard deviation. Two-factor analysis of variance followed by the Least Significant Difference post hoc test was used to compare the four groups to determine sex-related differences, and differences between individuals with DM and NGT. Correlation coefficients were analyzed using Pearson's correlation. $\mathrm{P}<0.05$ was considered to indicate a statistically significant difference.

\section{Results}

Baseline characteristics. Age and body mass index were comparable in the four groups (Table I). HbAlc, FPG and 2-h PG levels were significantly increased in premenopausal women and men with DM compared with in normoglycemic premenopausal women and men, respectively $(\mathrm{P}<0.05)$. Furthermore, women with NGT and DM had significantly higher estrogen levels than age- and glycemia-matched men $(\mathrm{P}<0.05)$. Although TC levels were increased in men with DM compared with in normoglycemic men, LDL and HDL cholesterol, TG, systolic blood pressure, diastolic blood pressure and high-sensitivity C-reactive protein levels were comparable between these groups.

Characterization of early EPCs. Prior to being cultured in EGM, the peripheral blood mononuclear cells suspended in the medium demonstrated a monocyte-like appearance. Following 7 days of culture, the peripheral blood mononuclear cells presented a typical 'spindle-shaped' appearance. Fluorescent immunocytochemistry was used to observe adherent cells that were stained for both DiI-acLDL and FITC-lectin (Fig. 1).

Number and activity of circulating EPCs in the four groups. As shown in Fig. 2A and B, the number of circulating EPCs among the four groups was evaluated using flow cytometric analysis and cell culture assay. Premenopausal women and men with DM exhibited a significantly decreased number of EPCs compared with the corresponding NGT patients $(\mathrm{P}<0.05)$. The male population with and without DM had a significantly lower number of EPCs compared with in the glycemia-matched female population $(\mathrm{P}<0.05)$. The two methods exhibited a similar trend when comparing the number of EPCs among the four groups. 
Table I. Clinical and biochemical characteristics.

\begin{tabular}{|c|c|c|c|c|}
\hline Characteristics & NGT women & Diabetic women & NGT men & Diabetic men \\
\hline No. & 20 & 20 & 20 & 20 \\
\hline Age (years) & $42.2 \pm 4.6$ & $42.7 \pm 4.8$ & $43.7 \pm 4.4$ & $44.3 \pm 4.0$ \\
\hline Height $(\mathrm{cm})$ & $162.0 \pm 5.9$ & $162.4 \pm 5.8$ & $167.4 \pm 6.0^{\mathrm{b}}$ & $168.1 \pm 6.0^{\mathrm{b}}$ \\
\hline Weight (kg) & $60.2 \pm 5.3$ & $62.0 \pm 5.8$ & $65.2 \pm 4.9^{\mathrm{b}}$ & $66.0 \pm 4.6^{\mathrm{b}}$ \\
\hline BMI $\left(\mathrm{kg} / \mathrm{cm}^{2}\right)$ & $23.0 \pm 1.9$ & $23.5 \pm 1.9$ & $23.3 \pm 1.4$ & $23.6 \pm 1.9$ \\
\hline Systolic blood pressure (mmHg) & $118.7 \pm 9.9$ & $114.7 \pm 9.5$ & $119.9 \pm 12.3$ & $116.4 \pm 10.6$ \\
\hline Diastolic blood pressure (mmHg) & $73.4 \pm 5.8$ & $71.5 \pm 5.1$ & $75.0 \pm 6.4$ & $72.4 \pm 5.8$ \\
\hline Heart rate (beats/min) & $75.4 \pm 9.0$ & $78.1 \pm 8.4$ & $80.7 \pm 7.7$ & $79.3 \pm 8.7$ \\
\hline AST (mmol/l) & $26.4 \pm 5.6$ & $25.2 \pm 5.5$ & $27.4 \pm 5.4$ & $28.2 \pm 4.6$ \\
\hline ALT (mmol/l) & $23.5 \pm 6.5$ & $22.2 \pm 5.2$ & $24.2 \pm 4.5$ & $24.4 \pm 4.7$ \\
\hline BUN (mmol/l) & $4.8 \pm 1.0$ & $4.5 \pm 0.9$ & $4.9 \pm 0.9$ & $4.6 \pm 0.8$ \\
\hline $\mathrm{Cr}(\mathrm{mmol} / \mathrm{l})$ & $60.5 \pm 10.6$ & $55.6 \pm 11.7$ & $61.1 \pm 12.3$ & $57.5 \pm 12.9$ \\
\hline LDL (mmol/l) & $2.8 \pm 0.3$ & $3.0 \pm 0.4$ & $2.9 \pm 0.3$ & $2.8 \pm 0.5$ \\
\hline $\mathrm{TC}(\mathrm{mmol} / \mathrm{l})$ & $4.7 \pm 0.4$ & $4.78 \pm 0.5$ & $4.85 \pm 0.3$ & $4.93 \pm 0.4^{\mathrm{a}}$ \\
\hline HDL (mmol/1) & $1.3 \pm 0.2$ & $1.3 \pm 0.2$ & $1.3 \pm 0.2$ & $1.3 \pm 0.2$ \\
\hline TG (mmol/l) & $1.5 \pm 0.2$ & $1.5 \pm 0.1$ & $1.5 \pm 0.2$ & $1.5 \pm 0.1$ \\
\hline FPG (mmol/l) & $4.7 \pm 0.7$ & $8.9 \pm 1.2^{\mathrm{a}}$ & $4.5 \pm 0.6$ & $8.7 \pm 1.1^{\mathrm{a}}$ \\
\hline 2-h PG ( mmol/l) & $6.3 \pm 0.7$ & $10.8 \pm 1.7^{\mathrm{a}}$ & $6.2 \pm 0.7$ & $10.3 \pm 1.6^{\mathrm{a}}$ \\
\hline HbA1c (\%) & $5.3 \pm 0.6$ & $8.5 \pm 1.5^{\mathrm{a}}$ & $5.2 \pm 0.6$ & $82 \pm 1.5^{\mathrm{a}}$ \\
\hline hsCRP (mmol/l) & $1.0 \pm 0.5$ & $1.1 \pm 0.6$ & $2.0 \pm 0.9$ & $1.8 \pm 0.9$ \\
\hline Estradiol (pmol/l) & $206.0 \pm 26.2$ & $216.5 \pm 37.0$ & $102.5 \pm 20.1^{b}$ & $108.1 \pm 19.9^{b}$ \\
\hline FMD $(\%)$ & $9.8 \pm 1.6$ & $8.3 \pm 1.2^{\mathrm{a}}$ & $9.1 \pm 1.9^{\mathrm{b}}$ & $7.2 \pm 1.2^{\mathrm{a}, \mathrm{b}}$ \\
\hline
\end{tabular}

2-h PG, 2 h post glucose; ALT, alanine aminotransferase; AST, aspartate transaminase; BMI, body mass index; BUN, blood urea nitrogen; Cr, creatinine; FMD, flow-mediated brachial artery dilatation; FPG, fasting plasma glucose; HbA1c, glycated hemoglobin; HDL, high-density lipoprotein; hsCRP, high-sensitivity C-reactive protein; LDL, low-density lipoprotein; NGT, normal glucose tolerance; TC, total cholesterol; TG, triglyeride. Data are presented as the means \pm standard deviation. ${ }^{\mathrm{a}} \mathrm{P}<0.05$ vs. NGT; ${ }^{\mathrm{b}} \mathrm{P}<0.05$ vs. premenopausal women.

The results demonstrated that the migratory and proliferative activity of EPCs was significantly decreased in premenopausal women and men with DM compared with in normoglycemic premenopausal women and men $(\mathrm{P}<0.05$; Fig. $3 \mathrm{~A}$ and $\mathrm{B})$. Furthermore, migratory and proliferative activity of EPCs was significantly lower in the male population with NGT or T2DM compared with in women with NGT and DM $(\mathrm{P}<0.05)$.

FMD, plasma NO, VEGF and GM-CSF levels in the four groups. Differences between the sexes with regards to FMD and plasma NO levels were detected, indicating that premenopausal women with NGT and DM exhibited significantly higher FMD and plasma NO levels compared with in the age- and glycemia-matched men ( $\mathrm{P}<0.05$; Table I and Fig. 4A). However, the plasma NO levels in premenopausal women with DM were significantly decreased compared with in the NGT group, which was similar to the difference between men with NGT and DM $(\mathrm{P}<0.05)$. Plasma VEGF and GM-CSF levels exhibited no significant differences between the groups (P>0.05; Fig. 4B and C).

NO, VEGF and GM-CSF secretion by EPCs in the four groups. The difference in NO secretion based on cultured EPCs between the NGT and DM groups was in line with that of plasma NO levels; NO secretion was significantly reduced in patients with T2DM compared with in the NGT group, and significantly reduced in the male population when compared with the female population $(\mathrm{P}<0.05$; Fig. $5 \mathrm{~A})$. However, the secretion of VEGF and GM-CSF from cultured EPCs was comparable among all of the groups ( $\mathrm{P}>0.05$; Fig. 5B and $\mathrm{C})$.

Correlation between FMD and circulating EPCs. A significant positive correlation was detected between the number of circulating EPCs, as evaluated by flow cytometry $(r=0.49, \mathrm{P}<0.05)$ and cell culture ( $\mathrm{r}=0.56, \mathrm{P}<0.05)$, and FMD (Fig. 6A and $\mathrm{B}$ ). Similarly, the migratory and proliferative activity of EPCs was significantly positively correlated with FMD ( $\mathrm{r}=0.64, \mathrm{P}<0.05$ and $r=0.57, \mathrm{P}<0.05$, respectively; Fig. $6 \mathrm{C}$ and $\mathrm{D})$.

Correlation between FMD and NO levels in the plasma and secreted by EPCs. In addition, the correlations between FMD and plasma NO levels, and between FMD and NO secretion by EPCs were analyzed. Both plasma NO levels and NO secretion by EPCs were significantly positively correlated with FMD $(\mathrm{r}=0.68, \mathrm{P}<0.05$ and $\mathrm{r}=0.51, \mathrm{P}<0.05$; Fig. $6 \mathrm{E}$ and $\mathrm{F})$.

\section{Discussion}

The present study demonstrated that the number and activity of circulating EPCs were lower, and endothelial function was 


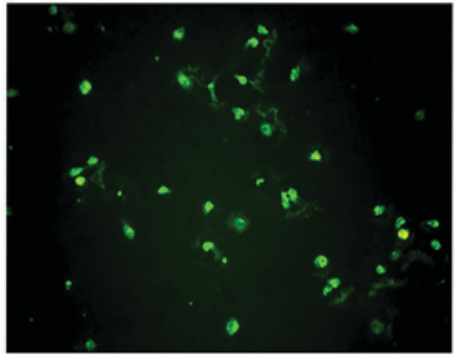

FITC-Lectin

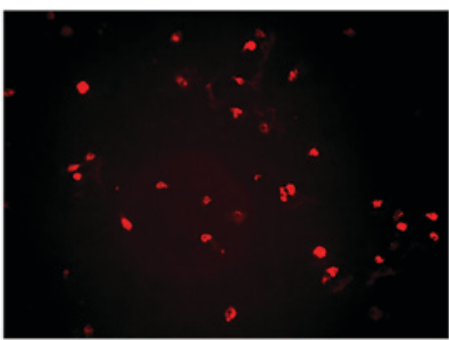

Dil-acLDL

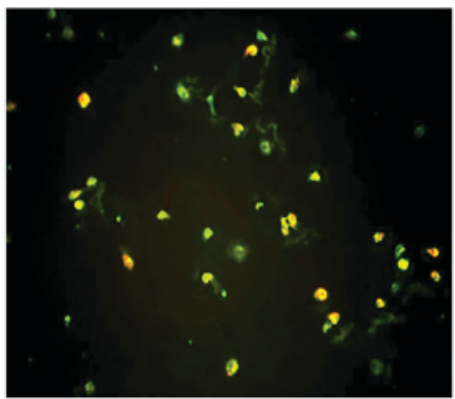

Merged

Figure 1. Characterization of cultured EPCs. Representative photographs of EPCs labeled with DiI-acLDL (red) and FITC-Lectin (green) following 7 days of incubation are presented, as well as a merged image. EPCs, endothelial progenitor cells; FITC, fluorescein isothiocyanate; acLDL, acetylated LDL low-density lipoprotein; DiI-acLDL, 1,1'-dioctadecyl-3,3,3',3'-tetramethylindo-carbocyanine perchlorate-labeled acetylated LDL.

impaired in premenopausal women with T2DM compared with in women with NGT, which was at least partly attributable to decreases in NO production. It was therefore hypothesized that attenuated endothelial function, which may be induced by impaired circulating EPCs, may be associated with an increased CVD risk, to a certain extent, in patients with DM. Differences between the sexes in the number and activity of EPCs were also observed both in the population with and without T2DM.

In the present study, DM was reported to impair the capability of endothelial cells to repair and it was suggested that it may lead to endothelial dysfunction in premenopausal women with DM, which was at least partly attributable to impaired NO production. These results provided novel evidence on the important role of endothelial protective interventions in premenopausal women with DM.

DM is widely regarded as an important risk factor for CVD pathogenesis, and contributes to cardiovascular morbidity and mortality (18). Even in premenopausal women with DM, cardiovascular risk is increased compared with in age-matched women without DM, thus suggesting that DM may increase the CVD risk in young premenopausal women due to certain underlying mechanisms (19). Previously, numerous studies indicated
A

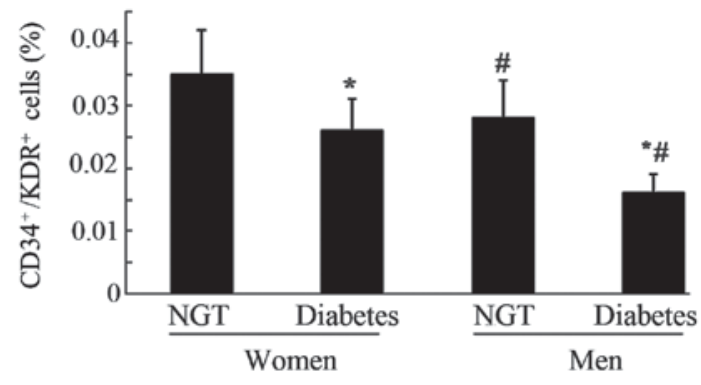

B

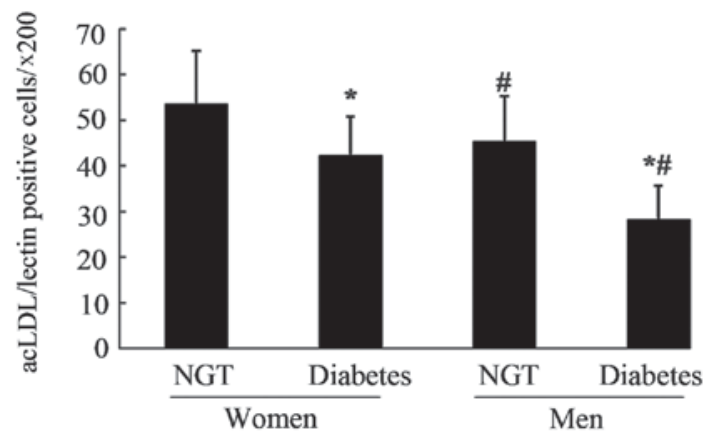

Figure 2. Number of circulating EPCs in the four groups. Circulating EPCS were evaluated by (A) flow cytometric analysis and (B) phase-contrast fluorescent microscopy; the number of circulating EPCs in men with NGT was decreased compared with in women with NGT (A, P=0.004; B, P=0.001) and significant reduction of the number of circulating EPCs was also observed in men with DM compared with premenopausal women with DM (A, P<0.0001; $\mathrm{B}, \mathrm{P}=0.002)$. The number of circulating EPCs in with women and men DM was decreased compared with in women and men with NGT (all $\mathrm{P}<0.0001$ ). Data are presented as the means \pm standard deviation. ${ }^{*} \mathrm{P}<0.05$ vs. NGT; ${ }^{\#} \mathrm{P}<0.05$ vs. premenopausal women. acLDL, acetylated LDL low-density lipoprotein; CD, cluster of differentiation; DM, diabetes mellitus; KDR, kinase-insert domain receptor; NGT, normal glucose tolerance; EPCs, endothelial progenitor cells.
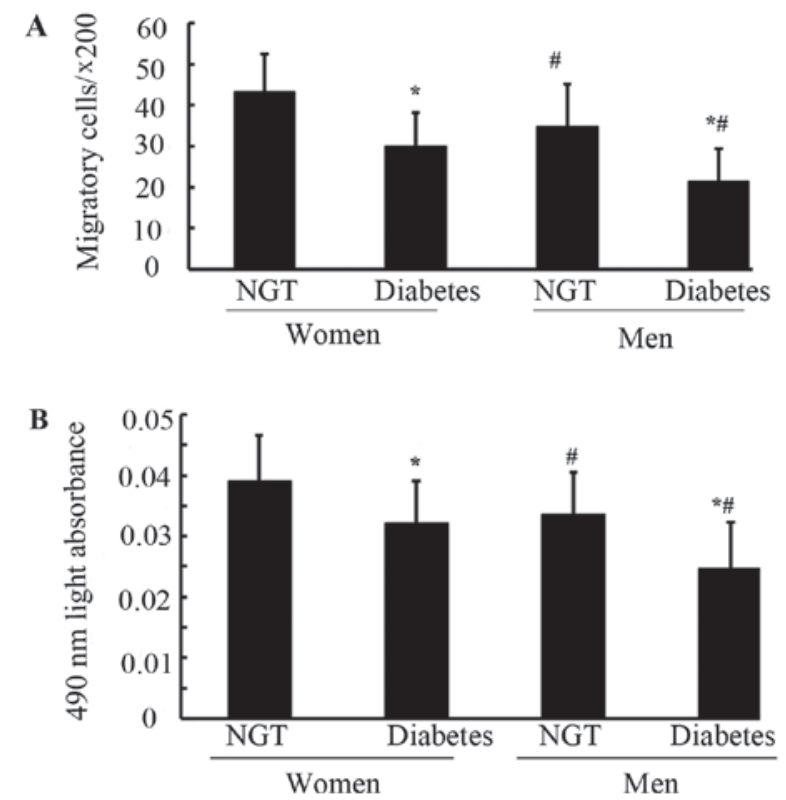

Figure 3. Activity of circulating EPCs in the four groups. (A) Migratory and (B) proliferative activities of circulating EPCs in men with NGT and DM were decreased compared with in premenopausal women with NGT (A, $\mathrm{P}=0.004 ; \mathrm{B}, \mathrm{P}=0.002)$ and $\mathrm{DM}(\mathrm{A}, \mathrm{P}=0.002 ; \mathrm{B}, \mathrm{P}<0.0001)$. The migratory and proliferative activities of circulating EPCs in women and men with DM were decreased compared with in women and men with NGT (all P<0.0001). Data are presented as the means \pm standard deviation. ${ }^{*} \mathrm{P}<0.05$ vs. NGT; ${ }^{\#} \mathrm{P}<0.05$ vs. premenopausal women. DM, diabetes mellitus; EPCs, endothelial progenitor cells; NGT, normal glucose tolerance. 

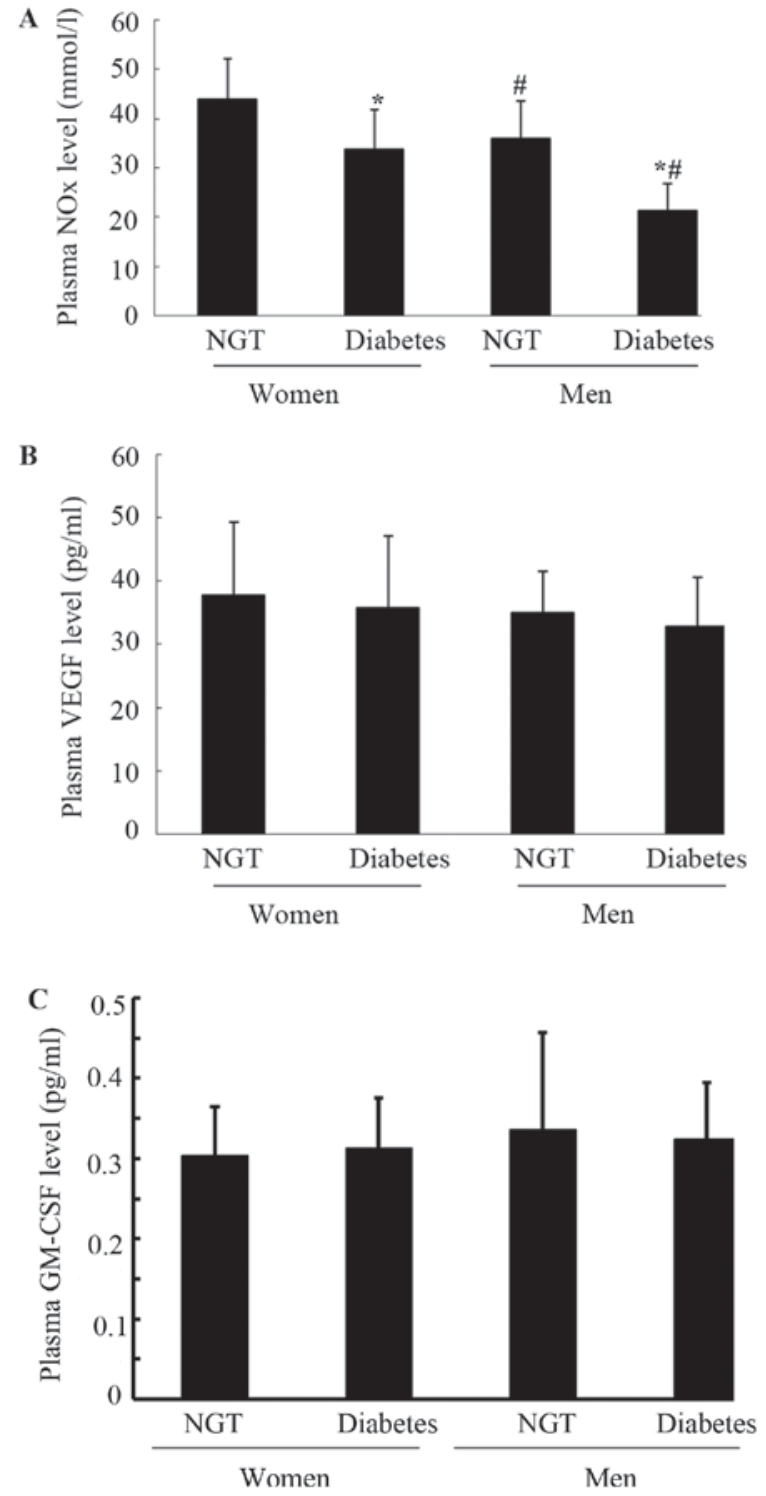

Figure 4. Plasma NO, VEGF and GM-CSF levels in the four groups. (A) Plasma NO levels in men with NGT and DM were decreased compared with those in premenopausal women with NGT and DM ( $\mathrm{P}=0.003$ and $\mathrm{P}=0.002$, respectively). Plasma NO levels in women and men with DM were decreased compared with in women and men with NGT (both $\mathrm{P}<0.0001$ ). (B) There was no significant difference in plasma VEGF levels between the four groups. (C) There was no significant difference in plasma GM-CSF levels between the four groups. Data are presented as the means \pm standard deviation. ${ }^{*} \mathrm{P}<0.05$ vs. NGT; ${ }^{\text {}} \mathrm{P}<0.05$ vs. premenopausal women. $\mathrm{DM}$, diabetes mellitus; GM-CSF, granulocyte macrophage-colony stimulating factor; NGT, normal glucose tolerance; NOx, nitric oxide; VEGF, vascular endothelial growth factor.

that the number and functional ability of circulating EPCs were reduced and weakened, due to the effects of impaired endothelial repair capacity during DM development or DM-associated vascular dysfunction $(11,13,14,31-33)$. The subjects in these studies were either patients with type $2 \mathrm{DM}$ and a mean age of $>50$ years old $(13,14)$ or patients with type $1 \mathrm{DM}$ and a mean age of $<30$ years old (31), as age has been reported to possibly influence the number of circulating EPCs (34). In prehypertensive premenopausal women, hyperglycemia weakens the preservation of the number and functional activity of EPCs and damages endothelial diastolic function (21). However,
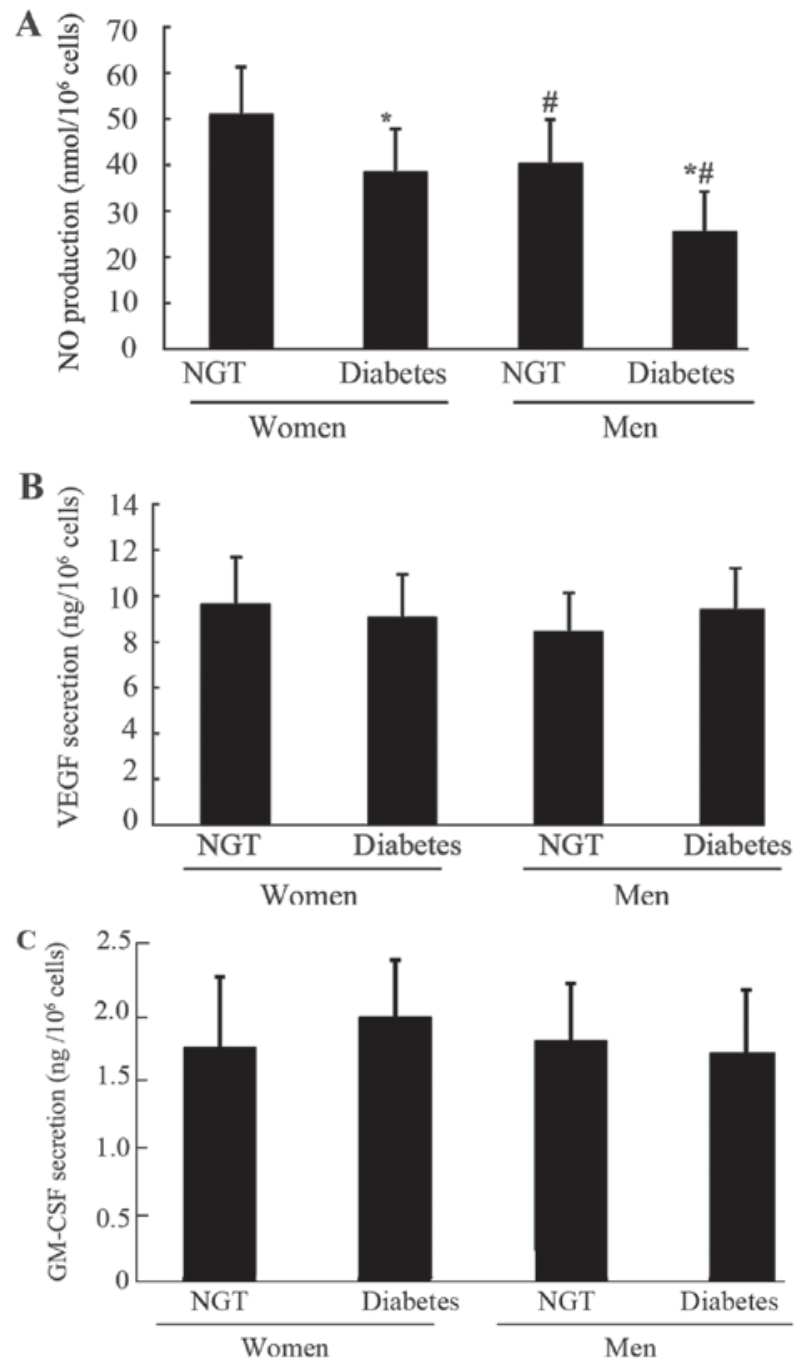

Figure 5. NO, VEGF and GM-CSF secretion by EPCs in the four groups. (A) NO secretion by EPCs in men with NGT and DM was decreased compared with in premenopausal women with NGT and DM $(\mathrm{P}=0.001$ and $\mathrm{P}<0.0001$, respectively). NO secretion by EPCs in women and men with DM was decreased compared with in women and men with NGT (both $\mathrm{P}<0.0001$ ). (B) There was no significant difference in VEGF secretion by EPCs between the four groups. (C) There was no significant difference in GM-CSF secretion by EPCs between the four groups. Data are presented as the means \pm standard deviation. "P<0.05 vs. NGT; ${ }^{~} \mathrm{P}<0.05$ vs. premenopausal women. DM, diabetes mellitus; EPCs, endothelial progenitor cells; GM-CSF, granulocyte macrophage colony stimulating factor; NGT, normal glucose tolerance; NO, nitric oxide; VEGF, vascular endothelial growth factor.

whether the impaired endothelial repair capability resulting from T2DM is present in the general premenopausal female population remains unclear. The present study detected a reduced number of circulating EPCs with impaired activity in premenopausal women with T2DM, but not in women without T2DM. FMD, an index used to assess endothelial function, was also decreased in these women, which was positively associated with the number and activity of EPCs. Therefore, it was hypothesized that the high CVD risk in premenopausal women with DM may at least partly result from suppressed endothelial repair capability; this finding differed from the results of our previous study, which indicated that prehypertensive premenopausal women possessed preserved endothelial repair capabilities (20). When compared with prehypertension, DM 

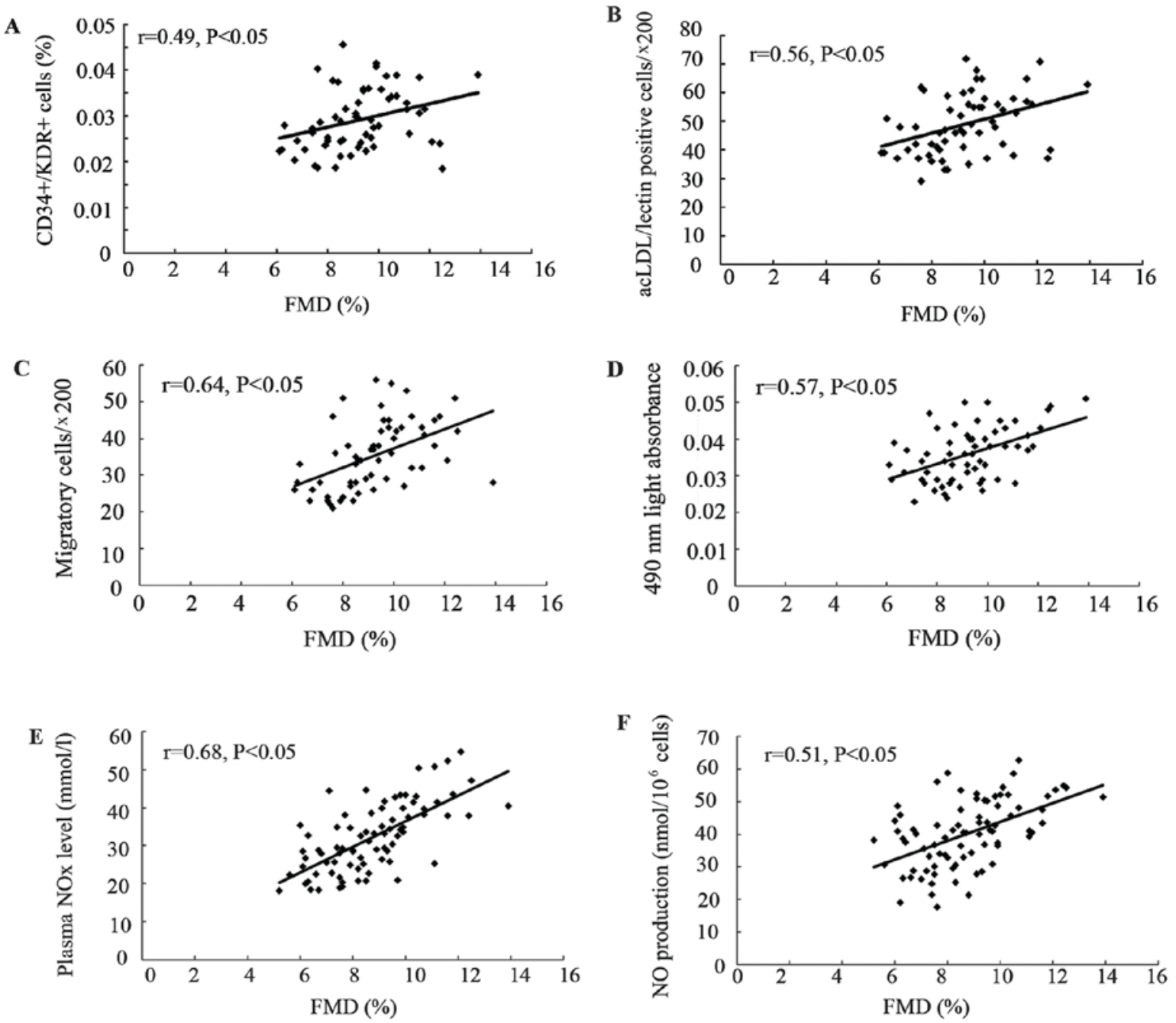

Figure 6. Correlation between FMD and circulating EPCs and NO levels. The number of circulating EPCs evaluated by (A) flow cytometry or by (B) cell culture was correlated with FMD. A correlation also existed between the (C) migratory and (D) proliferative activity of EPCs and FMD. (E) Plasma NO levels and (F) NO secretion by EPCs were correlated with FMD. LDL, low density lipoprotein; CD, cluster of differentiation; KDR, kinase-insert domain receptor; FMD, flow-mediated brachial artery dilatation; EPCs, endothelial progenitor cells; NO, nitric oxide.

hyperglycemia may have a more severe effect on endothelial repair capability and even on endothelial diastolic function. Therefore, intensive endothelial protective actions should be adopted in premenopausal women with DM.

In the male population of the present study, DM reduced the number, as well as the functional activity of circulating EPCs, which was consistent with the results in premenopausal women. Furthermore, sex-related differences in the number and activity of circulating EPCs were consistent in patients with and without DM, thus suggesting that the DM male population may be at risk for CVD due to dual impairment (low estrogen levels and DM) in their endothelial repair capability.

Although reductions in the number and functional activity of EPCs were observed, the mechanism underlying impairment or insufficient endothelial repair capability in patients with DM, and in the overall male population, remains unclear. NO, VEGF and GM-CSF are regarded as key factors in modulating the number and function of EPCs (22-25). In a previous study, it was revealed that NO bioavailability decreases in patients with DM due to impaired endothelial nitric oxide synthase (eNOS) activity, resulting from hyperglycemia (34). However, it remains to be determined as to whether reductions in the number and functional activity of circulating EPCs in premenopausal women with DM are correlated with alterations in NO, VEGF and GM-CSF. To investigate this, the plasma levels of NO, VEGF and GM-CSF were evaluated. Plasma NO levels were decreased in patients with DM, and differed between male and female populations. In addition, NO levels were positively correlated with FMD; these findings indicated that reduced systemic production of NO may lead to endothelial dysfunction in premenopausal women with DM and in male populations. Alterations in plasma VEGF and GM-CSF levels were not significant, indicating that these factors may not be associated with impaired endothelial repair capacity in patients with DM.

According to the results of the present study, in addition to plasma NO levels, reduced endogenous NO biosynthesis may contribute to the decreased number and activity of EPCs and endothelial diastolic dysfunction, both in premenopausal women with DM and in the overall male population. Endogenous NO biosynthesis serves a crucial role in maintaining the functional activity of EPCs (35). Chen et al (34) demonstrated that high glucose levels decreased eNOS, FoxO1 and Akt phosphorylation levels as well as levels of bioavailable NO in both early and late EPCs. The present study observed that, consistent with the 
alterations in plasma NO levels, NO production was reduced in premenopausal women with DM and in the overall male population compared with in premenopausal women with NGT and in the female population, respectively. Furthermore, NO production by EPCs and FMD were positively correlated. Therefore, these results suggested that efforts to enhance endogenous NO biosynthesis may be beneficial in preventing endothelial dysfunction.

Several mechanisms, which are involved in hyperglycemia, are associated with NO production by either vascular endothelium or EPCs. Advanced glycation end products have been reported to reduce NO production and eNOS expression (36), and hyperglycemia-activated protein kinase $\mathrm{C}$ increases eNOS gene expression and triggers eNOS uncoupling, thus leading to decreased bioavailability of NO (37). Hyperglycemia may also impair eNOS serine phosphorylation, leading to decreased EPC-derived NO production (34). NO is regarded as a crucial component in promoting mobilization, proliferation and migration of circulating EPCs, which promotes the number and activity of circulating EPCs (35). Therefore, it was inferred that DM-induced decreased NO production, either by the vascular endothelium or EPCs, may serve a role in hyperglycemia-impaired endothelial repair capability in the young population.

The present study provided several important guidelines for research regarding DM-induced vascular disease. Firstly, the results revealed that since premenopausal women with DM had impaired endothelial repair capability compared with women with NGT, and thus the corresponding intervention should be considered in a timely fashion. Since NO production may contribute to endothelial dysfunction, appropriate therapies, including statin usage, exercise and certain anti-hyperglycemic agents that increase NO production, should be considered in a clinical setting. Secondly, sex-related differences in circulating EPCs existed in the DM population, thus suggesting that men with DM may have difficulties in maintaining normal endothelial function; therefore, strict interventions to improve endothelial repair capacity should be implemented immediately. In addition, the mechanism underlying the differences between the sexes with regards to circulating EPCs remains to be clarified, particularly with regards to the role of estrogen in modulating the number and activity of circulating EPCs in a relatively young population. Another limitation associated the present study was that it was only conducted using data from Chinese subjects; therefore, these findings need to be confirmed in other populations.

In conclusion, the present study demonstrated that the number and activity of circulating EPCs in premenopausal women with DM were significantly lower compared with in women without DM, which was possibly associated with reduced NO production. Impaired endothelial repair capability in premenopausal women with DM may be an important mechanism underlying the increased relative risk of CVD. Furthermore, since sex-related differences were detected in circulating EPCs in the DM population, vascular protection should be carefully considered in men with DM.

\section{Acknowledgements}

We are grateful to all participants, research staff and faculty staff members who participated in the present study. We would also thank Professor Jinxin Zhang from the Department of Medical Statistic and Epidemiology, School of Public Health, Sun Yat-sen University (Guangzhou, China) for his contribution to statistical analysis.

\section{Funding}

The present study was financially supported by grants from the National Natural Scientific Foundation of China (grant nos. 81670220, 31270992 and 30800215), the project of Guangdong Province Science and Technology Plan (grant nos. 2015A020212013 and 2013B021800275), the Guangdong Natural Science Foundation (grant no. 2014A030313086), the Fundamental Research Funds for Central Universities in Sun Yat-Sen University (grant nos. 17ykzd18 and 13ykpy24), the Science and Technology Program of Guangzhou City (grant no. 201803010008), the International Scientific and Technological Cooperation project of Guangzhou Economic and Technological Development Zone (grant no. 2017GH13), the Industrial Technology Research and Development funding projects of Guangdong Province (grant no. 2014A020212436), and the Medical Scientific Research Foundation of Guangdong Province (grant no. A2015127).

\section{Availability of data and materials}

The datasets used and/or analyzed during the present study are available from the corresponding author on reasonable request.

\section{Authors' contributions}

ZY and HH designed the present study, supervised the writing of the manuscript and provided the final approval of the version to be published. JuL and DH designed the study, performed the experiments, wrote and revised the manuscript and provided final approval of the manuscript version for publication. HY, XA and ZR performed the experiments. JiL and $\mathrm{HZ}$ were involved the analysis and interpretation of data and contributed to the revision of the manuscript.

\section{Ethics approval and consent to participate}

The present study was approved by the ethics committee of the First Hospital of Chenzhou and Xiangya Hospital, Central South University (Changsha, China).

\section{Patient consent for publication}

Written informed consent was obtained from all participants.

\section{Competing interests}

The authors declare they have no competing interests.

\section{References}

1. Yang W, Lu J, Weng J, Jia W, Ji L, Xiao J, Shan Z, Liu J, Tian H, $\mathrm{Ji} Q$, et al: Prevalence of diabetes among Men and Women in China. N Engl J Med 362: 1090-1101, 2010.

2. Avogaro A, de Kreutzenberg SV and Fadini G: Endothelial dysfunction: Causes and consequences in patients with diabetes mellitus. Diabetes Res Clin Pract 82 (Suppl 2): S94-S101, 2008. 
3. Berezin AE: Endothelial progenitor cells dysfunction and impaired tissue reparation: The missed link in diabetes mellitus development. Diabetes Metab Syndr 11: 215-220, 2017.

4. Ali M, Mehmood A, Anjum MS, Tarrar MN, Khan SN and Riazuddin S: Diazoxide preconditioning of endothelia progenitor cells from streptozotocin-induced type 1 diabetic rats improves their ability to repair diabetic cardiomyopathy. Mol Cell. Biochem 410: 267-279, 2015.

5. Jin P, Li T, Li X, Shen X and Zhao Y: Suppression of oxidative stress in endothelial progenitor cells promotes angiogenesis and improves cardiac function following myocardial infarction in diabetic mice. Exp Ther Med 11: 2163-2170, 2016.

6. Aicher A, Zeiher AM and Dimmeler S: Mobilizing endothelial progenitor cells. Hypertension 45: 321-325, 2005.

7. Giannotti G, Doerries C, Mocharla PS, Mueller MF, Bahlmann $\mathrm{FH}$, Horvàth $\mathrm{T}$, Jiang $\mathrm{H}$, Sorrentino $\mathrm{SA}$, Steenken $\mathrm{N}$ Manes C, et al: Impaired endothelial repair capacity of early endothelial progenitor cells in prehypertension: Relation to endothelial dysfunction. Hypertension 55: 1389-1397, 2010.

8. Mandraffino G, Sardo MA, Riggio S, D'Ascola A, Loddo S, Alibrandi A, Saitta C, Imbalzano E, Mandraffino R, Venza M, et al: Smoke exposure and circulating progenitor cells: Evidence for modulation of antioxidant enzymes and cell count. Clin Biochem 43: 1436-1442, 2010.

9. Dong Y, Wu Y, Choi HC and Wang S: Diabetic endothelium dysfunction, cardiovascular complications, and therapeutics. J Diabetes Res 2016: 5349801, 2016.

10. Tsai TH, Chai HT, Sun CK, Yen CH, Leu S, Chen YL, Chung SY, Ko SF, Chang HW, Wu CJ and Yip HK: Obesity suppresses circulating level and function of endothelial progenitor cells and heart function. J Transl Med 10: 137, 2012.

11. Hörtenhuber T, Rami-Mehar B, Satler M, Nagl K, Höbaus C Höllerl F, Koppensteiner R, Schernthaner G, Schober E and Schernthaner GH: Endothelial progenitor cells are related to glycemic control in children with type 1 diabetes over time. Diabetes care 36: 1647-1653, 2013.

12. Fadini GP, Boscaro E, de Kreutzenberg S, Agostini C, Seeger F, Dimmeler S, Zeiher A, Tiengo A and Avogaro A: Time Course and Mechanisms of circulating progenitor cell reduction in the natural history of type 2 diabetes. Diabetes care 33: 1097-1102, 2010.

13. De Pascale MR, Bruzzese G, Crimi E, Grimaldi V, Liguori A, Brongo S, Barbieri M, Picascia A, Schiano C, Sommese L, et al: Severe type 2 diabetes induces reversible modifications of endothelial progenitor cells which are ameliorate by glycemic control Int J Stem Cells 9: 137-144, 2016.

14. Yue WS, Lau KK, Siu CW, Wang M, Yan GH, Yiu KH and Tse HF: Impact of glycemic control on circulating endothelial progenitor cells and arterial stiffness in patients with type 2 diabetes mellitus. Cardiovasc Diabetol 10: 113, 2011.

15. Sheetz MJ and King GL: Molecular understanding of hyperglycemia's adverse effects for diabetic complications. JAMA 288 2579-2588, 2002.

16. Cubbon RM, Mercer BN, Sengupta A and Kearney MT: Importance of insulin resistance to vascular repair and regeneration. Free Radical Bio Med 60: 246-263, 2013.

17. Giacco F and Brownlee M: Oxidative stress and diabetic complications. Circ Res 107: 1058-1070, 2010

18. Fox CS: Cardiovascular disease risk factors, type 2 diabetes mellitus, and the Framingham Heart Study. Trends Cardiovasc Med 20: 90-95, 2010

19. Garcia NH, Perez HA, Spence JD and Armando LJ: Risk of vascular disease in premenopausal women with diabetes mellitus. Clin Ther 36: 1924-1934, 2014

20. Zhen Y, Xiao S, Ren Z, Shen HW, Su H, Tang YB and Zeng H: Increased endothelial progenitor cells and nitric oxide in young prehypertensive women. J Clin Hypertens (Greenwich) 17: 298-305, 2015.

21. Zeng H, Jiang Y, Tang H, Ren Z, Zeng G and Yang Z: Abnormal phosphorylation of Tie2/Akt/eNOS signaling pathway and decreased number or function of circulating endothelial cells in prehypertensive premenopausal women with diabetes mellitus. BMC Endocr Disord 16: 13, 2016.
22. Yang Z, Wang JM, Chen L, Luo CF, Tang AL and Tao J: Acute exercise-induced nitric oxide production contributes to upregulation of circulating endothelial progenitor cells in healthy subjects. J Hum Hypertens 21: 452-460, 2007.

23. Bonafè F, Guarnieri C and Muscari C: Nitric oxide regulates multiple functions and fate of adult progenitor and stem cells. J Physiol Biochem 71: 141-153, 2015

24. Xue J, Du G, Shi J, Li Y, Yasutake M, Liu L, Li J, Kong Y, Wang S, Yun $\mathrm{F}$ and $\mathrm{Li} \mathrm{W}$ : Combined treatment with erythropoietin and granulocyte colony-stimulating factor enhances neovascularization and improves cardiac function after myocardial infarction. Chin Med J (Engl) 127: 1677-1683, 2014.

25. Shurygin MG, Shurygina IA, Dremina NN and Kanya OV: Endogenous progenitors as the source of cell material for ischemic damage repair in experimental myocardial infarction under conditions of changed concentration of vascular endothelial growth factor. Bull Exp Biol Med 158: 528-531, 2015.

26. Expert Committee on the Diagnosis and Classification of Diabetes Mellitus: Report of the expert committee on the diagnosis and classification of diabetes mellitus. Diabetes Care 26 (Suppl 1): S5-S20, 2003

27. Yang Z, Chen L, Su C, Xia WH, Wang Y, Wang JM, Chen F, Zhang YY, Wu F, Xu SY, et al: Impaired endothelial progenitor cell activity is associated with reduced arterial elasticity in patients with essential hypertension. Clin Exp Hypertens 32: 444-452, 2010

28. Yang Z, Tao J, Wang JM, Tu C, Xu MG, Wang Y and Pan SR: Shear stress contributes to t-PA mRNA expression in human endothelial progenitor cells and nonthrombogenic potential of small diameter artificial vessels. Biochem Biophys Res Commun 342: 577-584, 2006.

29. Yang Z, Xia WH, Zhang YY, Xu SY, Liu X, Zhang XY, Yu BB, Qiu YX and Tao J: Shear stress-induced activation of Tie2-dependent signaling pathway enhances reendothelialization capacity of early endothelial progenitor cells. J Mol Cell Cardiol 52: 1155-1163, 2012.

30. Corretti MC, Anderson TJ, Benjamin EJ, Celermajer D, Charbonneau F, Creager MA, Deanfield J, Drexler H, Gerhard-Herman M, Herrington D, et al: Guidelines for the ultrasound assessment of endothelial-dependent flow-mediated vasodilation of the brachial artery: A report of the International Brachial Artery Reactivity Task Force. J Am Coll Cardiol 39: 257-265, 2002.

31. Sibal L, Aldibbiat A, Agarwal SC, Mitchell G, Oates C, Razvi S, Weaver JU, Shaw JA and Home PD: Circulating endothelial progenitor cells, endothelial function, carotid intima-media thickness and circulating markers of endothelial dysfunction in people with type 1 diabetes without macrovascular disease or microalbuminuria. Diabetologia 52: 1464-1473, 2009.

32. Tsukada S, Masuda H, Jung SY, Yun J, Kang S, Kim DY, Park JH, Ji ST, Kwon SM and Asahara T: Impaired development and dysfunction of endothelial progenitor cells in type 2 diabetic mice. Diabetes Metab 43: 154-162, 2017.

33. Fadini GP, Sartore S, Agostini C and Avogaro A: Significance of endothelial progenitor cells in subjects with diabetes. Diabetes Care 30: 1305-1313, 2007.

34. Chen YH, Lin SJ, Lin FY, Wu TC, Tsao CR, Huang PH, Liu PL, Chen YL and Chen JW: High glucose impairs early and late endothelial progenitor cells by modifying nitric oxide-related but not oxidative stress-mediated mechanisms. Diabetes 56 1559-1568, 2007.

35. Aicher A, Heeschen C and Dimmeler S: The role of NOS3 in stem cell mobilization. Trends Mol Med 10: 421-425, 2004

36. Shen C, Li Q, Zhang YC, Ma G, Feng Y, Zhu Q, Dai Q, Chen Z, Yao Y, Chen L, et al: Advanced glycation endproducts increase EPC apoptosis and decrease nitric oxide release via MAPK pathways. Biomed Pharmacother 64: 35-43, 2010.

37. Cosentino F, Eto M, De Paolis P, van der Loo B, Bachschmid M, Ullrich V, Kouroedov A, Delli Gatti C, Joch H, Volpe M and Lüscher TF: High glucose causes upregulation of cyclooxygenase- 2 and alters prostanoid profile in human endothelial cells: Role of protein kinase $\mathrm{C}$ and reactive oxygen species. Circulation 107: 1017-1023, 2003. 